TUNCUR: Sequential Codes for Semiempirical Quantum-Chemical Calculations of Tunneling Current

\author{
Valentin A.Zayets and Elena F.Sheka \\ Russian Peoples Friendship University, Research Department, \\ ul.Ordjonikidze, 3, Moscow, 117302 Russia \\ sheka@icp.ac.ru
}

Keywords: tunneling current, STM image, quantum chemistry.

\begin{abstract}
TUNCUR codes are aimed at a coherent calculation of both equilibrated structures and electronic properties of a substrate and STM tip altogether with the tunneling current between them in the framework of the same computational scheme. Both objects are modeled by atomic clusters. Quantum chemical calculations in TUNCUR codes are provided by sequential programs CLUSTER-Z1 and CLUSTERZ2 that perform HF SCF calculations in the valence $s p$ - and $s p d$-basis, respectively. TUNCUR consist of three blocks that provide calculations of STM recordings in two modes of operation as well as computing local density of states. All calculations are performed over a grid of variable parameters.
\end{abstract}

\title{
1.Introduction
}

Since its invention [1], scanning tunneling microscopy (STM) has proven to be a powerful technique in viewing the surface structure of various systems with atomic resolution. In this technique, a small bias voltage $V$ is applied between a sample and an "atomically sharp" tip, which yields a tunneling current $I$ at typical tip-to-surface separation of several angstroms. Depending on the polarity of $V$, the tunneling occurs by transfer of electrons either from occupied states of the sample into unoccupied states of the tip, or from the tip to the sample. In the topographic mode (I=const mode) of STM operation [1], a feedback mechanism changes the tip-to-surface separation in order to keep the tunneling current constant. The STM image is given by recording the distance changes during a scan of the surface. In the alternative currentimaging mode ( $H=$ const mode) [2], an STM image is constituted by recording the tunneling current, as the tip scans the surface at a constant distance. No method has not been so powerful as SMT in observing a direct image of a surface on an atomic scale. However, numerous STM applications have revealed that STM does not necessarily image the atomic structure of the surface as a naïve ball model, but represents rather very delicate features of the surface electronic states (see $[3,4]$ and references there in). That is why STM so greatly needs a thorough theoretical analysis and/or calculations to decode profound information from the experimental images, as no other conventional method of the surface characterization such as electron microscopy or low-energy electron diffraction. 
A powerful theoretical and computational approach to the problem has been elaborated by a group of Japanese authors (starting with the first publication [5], a full list of references can be found in [3]) that has introduced atomistic structure of both surface and tip in the electron tunneling. The approach was based on the firstprincipal theory of STM and was implemented by using the first-principal local density functional (LDA) computational technique when applying to graphite, reconstructed $\mathrm{Si}(100)$, and $\mathrm{Si}(111) \sqrt{3} \times \sqrt{3}-\mathrm{Ag}$ surfaces (see [6] and references therein). A joint considering of the sample and tip on the same footing has resulted in a very good consistence with experiment, on the one hand, and has supplied obtaining reliable information concerning electronic structure of the sample, on the other. The stress in decoding STM images has shifted therefore to the problem of calculation of suitable clusters, large enough to be sufficient to reproduce the studied surface reliably.

The first attempt to substitute a DFT technique by more time-consuming semiempirical one has concerned the application of MINDO3 approach to the case [7]. Applying to $\mathrm{Si}(100)$ and $\mathrm{Si}(100)-\mathrm{H}$ surfaces, it occurred to be rather successful. Still there have been a few more attempts to consider STM images computationally, but no efficient program, particularly devoted to reliable and efficient permanent job in the STM image field has not been known. This paper presents computational codes to be efficient for the job.

\section{Computational algorithm}

Following to $[5,8]$, in terms of Bardeen's perturbation formalism the tunnel current in the LCAO representation can be expressed as

$$
\begin{aligned}
I= & \frac{2 \pi e}{\hbar} \int d E[f(E)-f(E-e V)] \sum_{i i^{\prime}} \sum_{p p^{\prime}} \sum_{j j^{\prime}} \sum_{q q^{\prime}} G_{i p i^{\prime} p^{\prime}}^{S}(E) \\
& \times G_{j q j^{\prime} q^{\prime}}^{T}(E-e V) J_{i p j q}(R) J_{i^{\prime} p^{\prime} j^{\prime} q^{\prime}}^{*}(R),
\end{aligned}
$$

where the tunnel matrix element $J_{i p j q}$ is given by

$$
J_{i p j q}(R)=\int_{\Omega_{T}} d r \chi_{p}^{*}\left(r-R-R_{i}\right) V^{T}(r) \phi_{q}\left(r-R_{j}\right),
$$

with the atomic basis function for the surface $\chi_{p}$ and for the tip $\phi_{q}$, respectively [5]. $G_{i p i^{\prime} p^{\prime}}^{S}$ and $G_{j q j^{\prime} q^{\prime}}^{T}$ are the LCAO representation of the surface and the tip Green's functions and expressed by

$$
\begin{aligned}
& G_{i p i^{\prime} p^{\prime}}^{S}(E)=\sum_{\mu} C_{\mu, i p}^{S} C_{\mu, i^{\prime} p^{\prime}}^{S *} \delta\left(E-E_{\mu}\right), \\
& G_{j q j^{\prime} q^{\prime}}^{T}(E)=\sum_{v} C_{v, j q}^{T} C_{\nu, j^{\prime} q^{\prime}}^{T *} \delta\left(E-E_{v}\right),
\end{aligned}
$$


respectively. Here $C_{\mu, i p}^{S}$ and $E_{\mu}\left(C_{v, j q}^{T}\right.$ and $\left.E_{v}\right)$ are the coefficient of the eigen state and its energy for the surface (tip). As shown [5], $G_{i p i^{\prime} p^{\prime}}^{S}(E)$ and $G_{j q j^{\prime} q^{\prime}}^{T}(E)$ do not depend on the relative geometry between the tip and the surface, thus they can be calculated only once. In Ex.(1) $e$ denotes electron charge, $\hbar$ is Plank's constant, $f(E)$ is the Fermi function $f(E)=\left[\exp \left(\left(E-E_{F}\right) / k T\right)+1\right]^{-1}$, where $k, T$, and $E_{F}$ are Bolzman constant, absolute temperature and Fermi energy, respectively. Indices $i, p$, $i^{\prime}, p^{\prime}$ are related to the surface (S) while those $j, q, j^{\prime}, q^{\prime}$ matches the tip. Indices $p$ and $p^{\prime}\left(q\right.$ and $\left.q^{\prime}\right)$ number the surface (tip) atom (index coincidence is possible) while $i$ and $i$ ' (correspondingly $j$ and $j^{\prime}$ ) number atom orbitals with $i \in p(j \in q)$ and $i^{\prime} \in p^{\prime}$ $\left(j^{\prime} \in q^{\prime}\right)$. Summation in (3) and (4) depends on the polarity of the sample bias voltage $V$ :

a) at $V>0$, summation over $\mu$ covers vacant orbitals of the surface while summation over $v$ is carried out over occupied orbitals of the tip; electron tunneling from the tip into the surface takes place;

b) at $V<0$, summations over $\mu$ and $v$ interchange and the electron tunneling from the surface into the tip is observed.

To make the calculated results for a cluster limited in size to be sufficient for real systems, we introduce Lorentzian functions replacing every delta function peak not only of the discrete system of the tip as in $[5,8]$ but of the surface as well, so that

$$
\begin{aligned}
& G_{i p i^{\prime} p^{\prime}}^{S}(E)=\sum_{\mu} C_{\mu, i p}^{S} C_{\mu, i^{\prime} p^{\prime}}^{S *} \frac{\Delta / \pi}{\left(E-E_{\mu}\right)^{2}+\Delta^{2}}, \\
& G_{i p i^{\prime} p^{\prime}}^{S}(E)=\sum_{v} C_{v, i p}^{S} C_{v, i^{\prime} p^{\prime}}^{S *} \frac{\Delta / \pi}{\left(E-E_{v}\right)^{2}+\Delta^{2}} .
\end{aligned}
$$

The width $\Delta$ in Ex.(6) has been naturally chosen in [5,8] as the d-band width of the tip metal of $\sim 1 \mathrm{eV}$. Hereinafter this value has been used in both Exs. (5) and (6). As checked, calculated results remained practically unchanged when the value changes by 0.5 or $2 \mathrm{eV}$.

All the geometrical information between tip and the surface as well as the relative orbital matching conditions are solely included in the tunnel matrix element $J_{i p j q}(R)$. As shown in $[5,8]$, the matrix element has the form

$$
J_{i p j q}(R)=\int_{\Omega_{T}} d r \chi_{p}^{*}\left(\left|r-R-R_{i}\right|\right) V^{T}(r) \phi_{q}\left(\left|r-R_{j}\right|\right)
$$

and similarly

$$
J_{i^{\prime} j^{\prime} q^{\prime}}^{*}(R)=\int_{\Omega_{T}} d r \chi_{p^{\prime}}\left(\left|r-R-R_{i^{\prime}}\right|\right) V^{T}(r) \phi_{q^{\prime}}^{*}\left(\left|r-R_{j^{\prime}}\right|\right) .
$$

Here $\chi_{p}$ and $\phi_{q}$ are basic AOs of the surface and the tip, respectively, in the valence "basis of the Slatter type. Fig.1 illustrates a scheme of the tunnel current determination by summing loops over all the indices. 


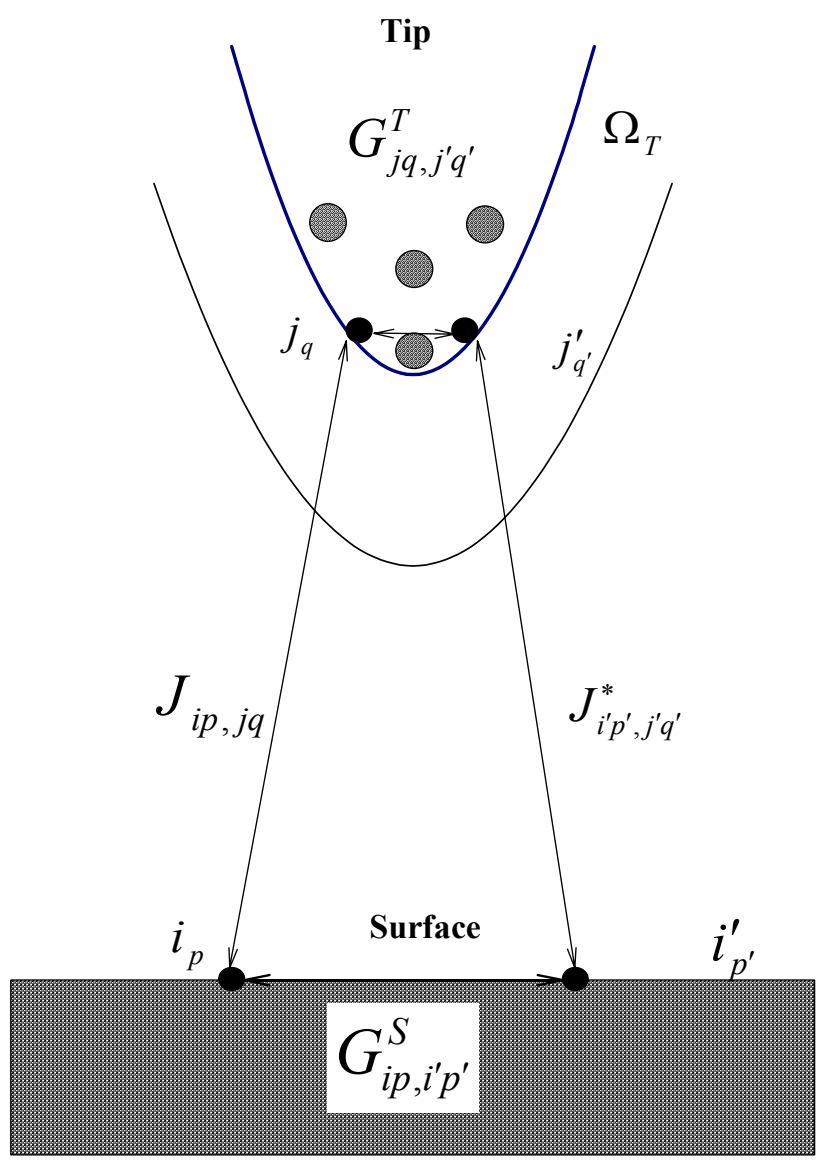

If $r=z \in Z_{1}, Z_{2}$, then

$V^{T}(r)=(z-Z O Z) \operatorname{tg} \varphi+C$,

where $C=-16.8 \mathrm{eV}$. Otherwise,

$V^{T}(r)=\left\{\begin{array}{lc}-P O T 0+C, & z<Z_{1} \\ \hline P O T 0+C, & z>Z_{2} .\end{array}\right.$
Figure 1. Construction of the tunnel current in the LCAO representation

\section{Calculation details}

\subsection{Tip potential $V^{T}$}

Tip potential $V^{T}(r)$ in Exs. \{7) and (8) is equal to the total potential on the tip side of the gap region between the tip and the surface with the vanishing potential on the surface side. The potential is of a piecewise form and is presented in Fig.2 alongside with parameters used for its determination in the TUNCUR code. The internal coordinate $r$ is determined in the tip region $\Omega_{T}$ with reference to a certain point $R$.

The potential parameters $C, R M A X, A L P H, X L E N$ should be chosen by providing a tunnel current of $p A$ in magnitude at the surface-tip distance of several angstroms. As occurred, the empirical procedure, suggested for the tip potential description, smoothes the problem of the wave function tails [3] to a great extent.

\subsection{Integration technique over $r$}

When integrating in (7) and (8), the tip is kept in a rectangle box with boundaries over $X$ as $X M I N=X L-R M A X S$ and $X M A X=X R+R M A X S$, where $X L$ and $X R$ are $X$ coordinates of terminal atoms of the tip. Similarly boundaries are determined for $Y$ and $Z$ coordinates. The space $\Omega_{T}$ is divided into $N_{x}, N_{y}, N_{z}$ rectangle cells. The integration is carried out over the cells analogously to one-dimensional integration 
over rectangle boxes. Wave functions $\chi(r)$ and $\phi(r)$ are calculated in the cell centers. Usually, $N=50$ for each coordinates is fully enough. Integration limits are regulated by $R M A X S$ within the space $\Omega_{T}$.

\subsection{TUNCUR calculation modes}

A general scheme of calculations is presented in Fig. 3. Those are started with a quantum chemical (QCh) simulation of studied surface and tip. Any QCh tool can be used on this stage if only provide well optimized geometry of both systems as well as sets of eigen states and eigen values. In a particular case of Fig.3, sequential codes of

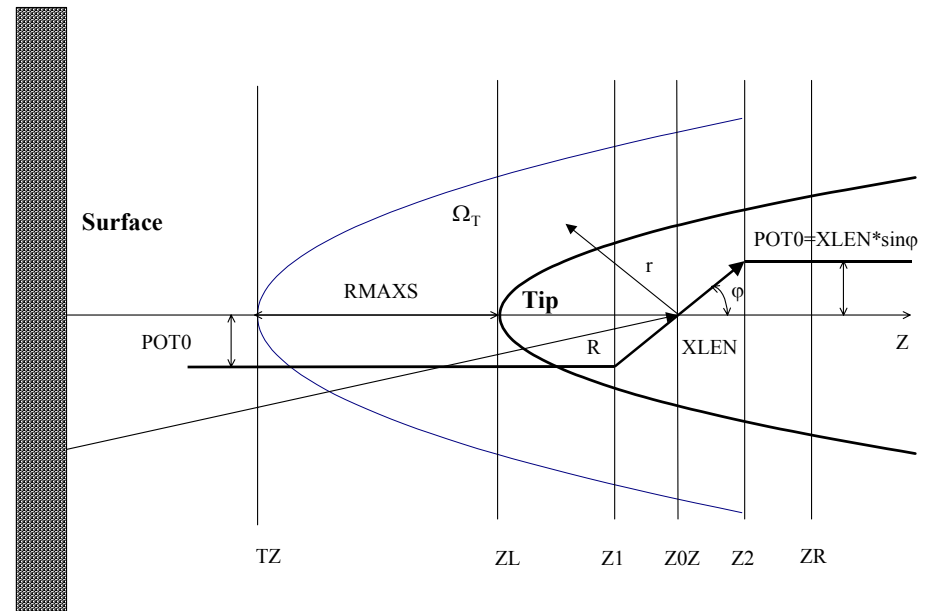

Figure 2. Space of $z$ coordinates needed to determine $V^{T}(r)$.

ZL denotes the tip end. ZR is a geometrical center of the tip.

$\mathrm{TZ}=\mathrm{ZL}-\mathrm{RMAX}, \mathrm{RMAX}$

$\mathrm{ZOZ}=\mathrm{TZ}+(\mathrm{ZR}-\mathrm{TZ}) \mathrm{ALPH}$

$\mathrm{Z} 1=\mathrm{ZOZ}-\mathrm{XLEN} * \cos \varphi$,

$\mathrm{Z} 2=\mathrm{ZOZ}+\mathrm{XLEN} * \sin \varphi$

$\mathrm{POT} 0=\mathrm{XLEN} * \sin \varphi$.

two QCh semi-empirical programs CLUSTER-Z1 [9] and CLUSTER-Z2 [10] do the job for large model clusters. Two files surf.in and tip.in with needed data are created on the basis of calculated results that form the first TUNCUR input file jobcurr. dat

\section{jobcurr.dat}

jobname surf tip

The second TUNCUR input file jobname.05 contains all necessary information concerning calculations within the framework of the TUNCUR codes without regarding which calculation mode is in use. The first line of the file concerns timing and restarting $(M I D D L E)$ of the job. Input information related to the sample bias voltage (VOLT, in V), parameter $\triangle$ in Exs. (5) and (6) (DELTA, in eV), temperature $(T$, in $\mathrm{K})$ and the Fermi energy index (EFERMI) are given in line 2. When EFERMI = 0.0 , the HOMO orbital energy is input as the Fermi one. The latter can be input directly from the screen as well just replacing EFERMI by the energy value in $\mathrm{eV}$. Line 3 contains integrating variable and the number of points for integrating over Sympson. Parameters of piecewise potential $V^{T}(r)$ are given in lines 4,5 , and 8 (see text). Figures, given in the file, are related to a four-atom Ti tip described below. Numbers of points when integrating over the tip are given in line 6 . Line 7 presents the starting geometry for scanning. It is recommended to locate the surface in the $Z=0$ plane so that $D X$ and $D Y$ determine $X$ and $Y$ coordinates of the point on the surface while $D Z$ shows the displacement of the tip along $Z$-direction with respect to the geometry given in files surf.in and tip.in. Line 9 presents boundary conditions for the 
grid covering the surface in the $X Y$ space. $Q Z 1$ and $Q Z 2$ in line 10 mark limits of the tip displacement for searching a preset value of the tunnel current when calculating over grid. TOL prescribes the tolerance when calculating the tip displacement in the topographic mode of operation. $N Q X$ and $N Q Y$ in line 11 determine the numbers of points over $X$ and $Y$ axes of the grid that coincide with the relevant axes of the surface.

\section{CLUSTER-Z1}

(QCh calculation in the valence $s p$-basis)

\section{CLUSTER-Z2}

(QCh calculation in the valence spdbasis)

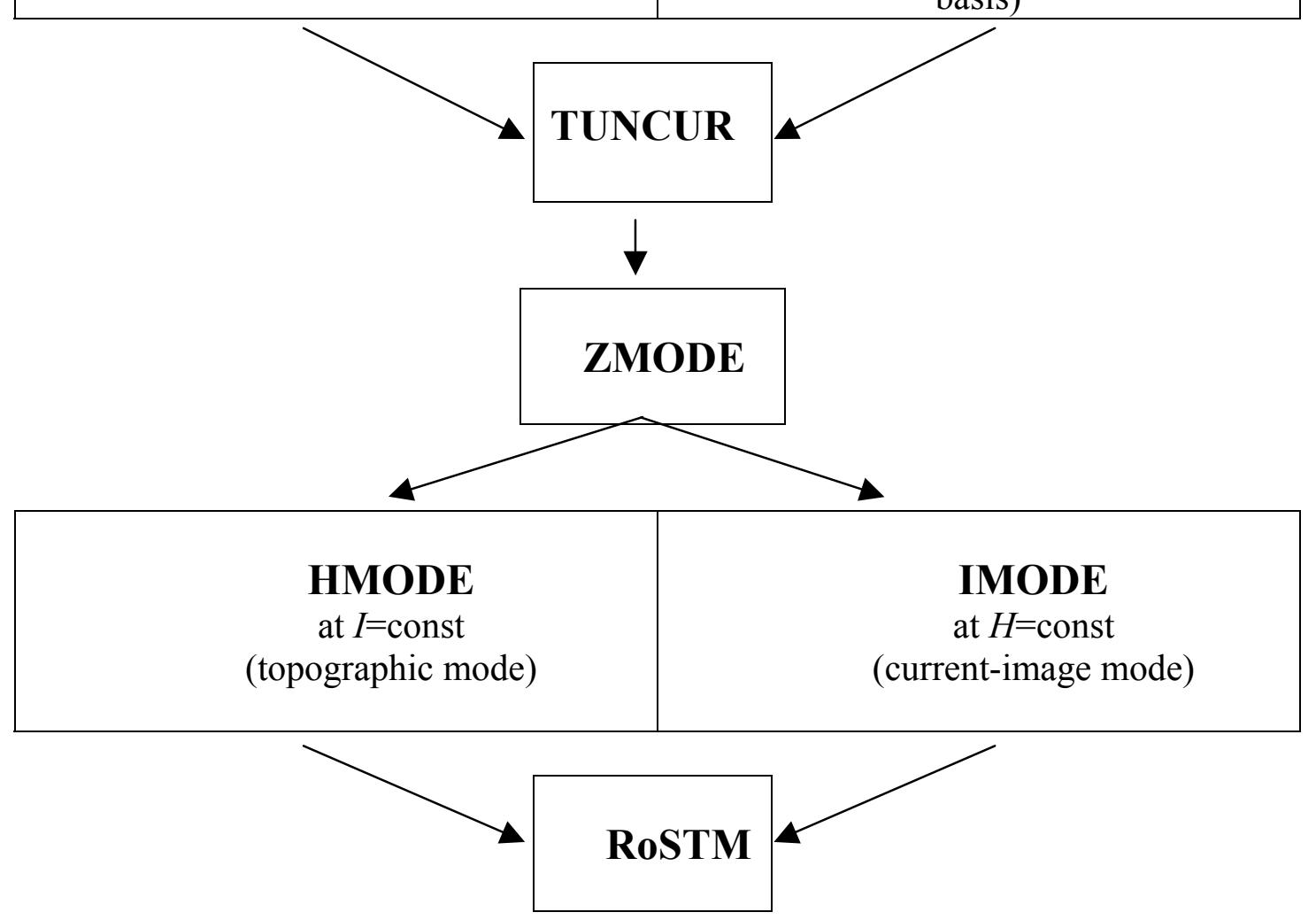

Figure 3. A general scheme of the tunnel current calculations.

Table 1. The main input file of the TUNCUR codes

\begin{tabular}{|c|c|c|c|c|c|c|c|}
\hline \multicolumn{8}{|c|}{ jobname. 05} \\
\hline 1 & 07 & 1830 & * & 1. & $\star 1800.0$ & * & TLIMIT , SFDFP, TDUMP , MIDDLE \\
\hline 2 & & -1.0 & $\star$ & 1 . & * 273.15 & \multirow[t]{2}{*}{0.0} & VOLT , DELTA, T , EFERMI \\
\hline 3 & & 10.0 & * & 10.0 & $\star 0200 \mathrm{~F}$ & & EDOWN , EUP , NEINT \\
\hline 4 & & 1.0 & * & 1.0 & 1.0 & \multirow{3}{*}{0.7} & $\mathrm{XL} 1, \mathrm{YL} 1, \mathrm{ZL} 1$ \\
\hline 5 & & 1.0 & * & 1.0 & 1.0 & & XM1 , YM1 , ZM1 , RMAXS \\
\hline 6 & & \multicolumn{2}{|c|}{$0050 *$} & $0050 *$ & $0050 *$ & & NXINT, NYINT, NZINT \\
\hline 7 & & -2.500 & * & 6.700 & -1.280 & * & $\mathrm{DX}, \mathrm{DY}, \mathrm{DZ}$ \\
\hline 8 & & 1.5 & * & 0.5 & 1.2 & $\star-55.0$ & XLEN , ALPH , C , ANGLE \\
\hline 9 & & -2.500 & * & 6.700 & -2.140 & $\star \quad+8.200$ & QLX , QRX, QLY, QRY \\
\hline 10 & & 0.4 & * & 0.4 & 0.0011 & $1 *$ & $\mathrm{QZ1}, \mathrm{QZ2}$, TOL \\
\hline 11 & & 0016 & & 0018 & & * & NQX, NQY \\
\hline
\end{tabular}


The ZMODE routine is used when calculations are performed for a fixed point at the surface ( $N Q X$ and $N Q Y$ are equal to 1) whose coordinates $D X$ and $D Y$ are shown in line 7 of the file. The tip position is given by $D Z$ and can be changed either in file jobname. 05 or automatically by inputting an additional service file. HMODE and IMODE programs of the TUNCUR codes provide calculations over the grid whose parameters are given in file jobname. 05. In the former case the standard program ZEROIN [11] is used when looking for a root of a non-linear equation with one variable.

All routines within the above QCh and TUNCUR codes are written on FORTRAN-90 programming language and run on both Windows and LINUX (UNIX) platforms without any changes in the FORTRAN codes. The latter was checked by using two FORTRAN-90 compilers, namely, Microsoft-Intel Compack and NAG-95 in the mode of the maximum degree of optimization.

\section{4. $\rho_{S T M}$ calculations}

The original Tersoff-Hamann theory of STM [12] in its simple version that uses a simplified model of the spherical tip by including only the s-orbitals, yields to a well known expression of the tunnel current for the weak bias (tip) case,

$$
I(R)=V \rho\left(R, E_{F}\right)
$$

where $\rho\left(R, E_{F}\right)$ is the local density of states (LDOS) of the surface at the center position $\mathrm{R}$ of the spherical tip and at the Fermi level $E_{F}$. The important feature of Eq.(11) is that the tunnel current is determined by the information carried by the surface electronic states as a system separated from the tip. This finding has been supposed for the analysis of most of the experimental data [4]. When tunnel current is not recorded experimentally but computed, the relation opens a possibility of checking calculated results when both quantities $I$ and $\rho\left(R, E_{F}\right)$ are calculated on the same computational footing. The quantity $\rho\left(R, E_{F}\right)$ is better to present as $\rho_{S T M}(R, V)$ that according to [13] under certain conditions can be expressed as

$$
\rho_{S T M}(R, V)=\int_{E_{F}-V}^{E_{F}} d E \rho(R, E)
$$

where

$\rho(R, E) \equiv \sum_{\mu}\left|\psi_{\mu}(R)\right|^{2} \delta\left(E_{\mu}-E\right)$

Here $\rho(R, E)$, as previously, is LDOS at the center of curvature of the tip $R$ and $\psi_{\mu}(R)$ are the electron eigen states of the unperturbed surface with corresponding energy $E_{\mu}$. The electron energies and wave functions are the same as those entering the tunnel current calculations in the previous sections. As shown [13], the calculated $\rho_{S T M}(R, V)$ fit experimentally observed STM images quite well. Basing on this 
finding, program RoSTM is involved in the TUNCUR to complete the tunnel current calculation by internal checking.

\section{Model calculations}

A model system, suggested for the codes approbation, was chosen by meeting the following requirements. It should be planar $(Z=0$ plane) and characterized by a distinctly seen atomic pattern. At the same time the model should be rather large in size but should not contain too many atoms for the analysis to be less timeconsuming. We have chosen a planar configuration of nine benzene rings shown in Fig.4. It consists of 30 carbon atoms and 14 hydrogen terminators and looks like a one-layer structure of graphite. Rows of carbon atoms are strictly aligned along $X$ and $Y$-direction that are directions of scanning over grid. However, the model cannot be regarded as that one of the graphite surface due to well known peculiarities of the latter caused by the underneath carbon atoms layers $[6,13]$. Nevertheless, thus selected model surface is preferable when demonstrating the computational tool facilities without entering the depth of discussion of the available experimental features. The latter often are quite spurious, depending on particular experimental conditions. At the same time the model is quite sufficient to make clear how much the calculated data are reasonable from the experimental viewpoint. A cluster of four

Ti atoms of symmetry $T_{d}$ simulated the tip.

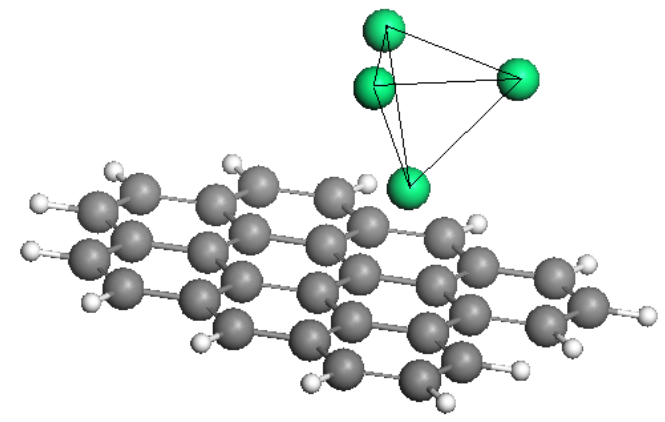

Figure 4. Optimized geometry of the "surface-tip" system. Surface-tip distance 3.20A.

QCh calculations have been
performed by using CLUSTER-Z2
software based on the NDDO-WF approximation [14-17] that allows Hartree-

Fock SCF calculations in the valence spd-basis (see detailed description of the calculation algorithm in [18]). Parameters for Ti atom are given elsewhere [19]. The structure of both the "surface" and "tip" was fully optimized. Putting together, the surface+tip system is shown in Fig.4. Parameters of the tip potential $V^{T}(r)$ can be written from the above file jobname. 05.

\section{1. $Z$-dependence of the tunnel current}

Shown in Fig.5 is Z-dependence of the tunnel current between the model surface and tip at two polarities of the surface bias voltage of $1 \mathrm{~V}$ by value. The tip is settled over the surface just so that its apex is situated exactly $2 \mathrm{~A}$ above surface atom number 20 . As seen from the figure, the current and voltage are of the same sign that is consistent with relation (11). At both polarities the current depends exponentially from the tip displacement that is particularly evident for linearly dependent the current logarithmic values. The current at the negative bias is less than that for the positive one and its values are of tens pA at surface-tip distance of $2 \mathrm{~A}$. Taking together, the obtained results fit well experimental data generally obtained (see for instance [20]). 


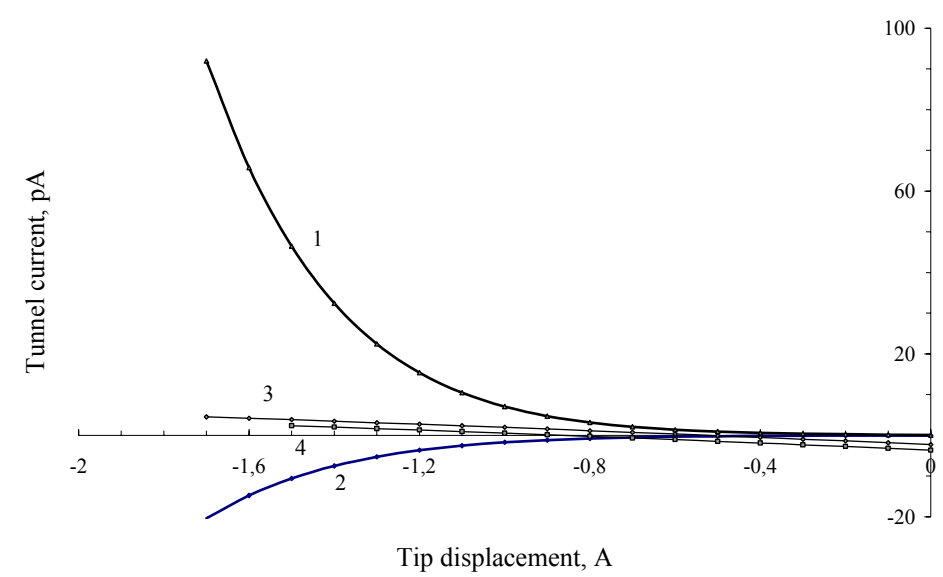

Figure 5. $Z$-dependence of the tunnel current $I$ (curves 1 and 2) and its logarithm $(\ln I)$ (curves 3 and 4 ) at the surface bias voltage $V=-1 \mathrm{~V}(1$ and 3 ) and $V=+1 \mathrm{~V}(2$ and 4$)$ for the model "surface+tip" system. ZMODE program in action.

Data presented in Table 2 show the contribution of both tip and surface atoms into the current. The maximum value in both cases is set to 100.0. As seen from the table, a relative contribution of the tip apex prevails $99 \%$ that is consistent with similar results of Tsukada et al [3]. Quite locally contributions are distributed over surface atoms as well. Obviously, the distribution of the atomic contributions will depend on the spatial resolution of an STM in practice as well as on the grid increment in the calculations.

Table 2.

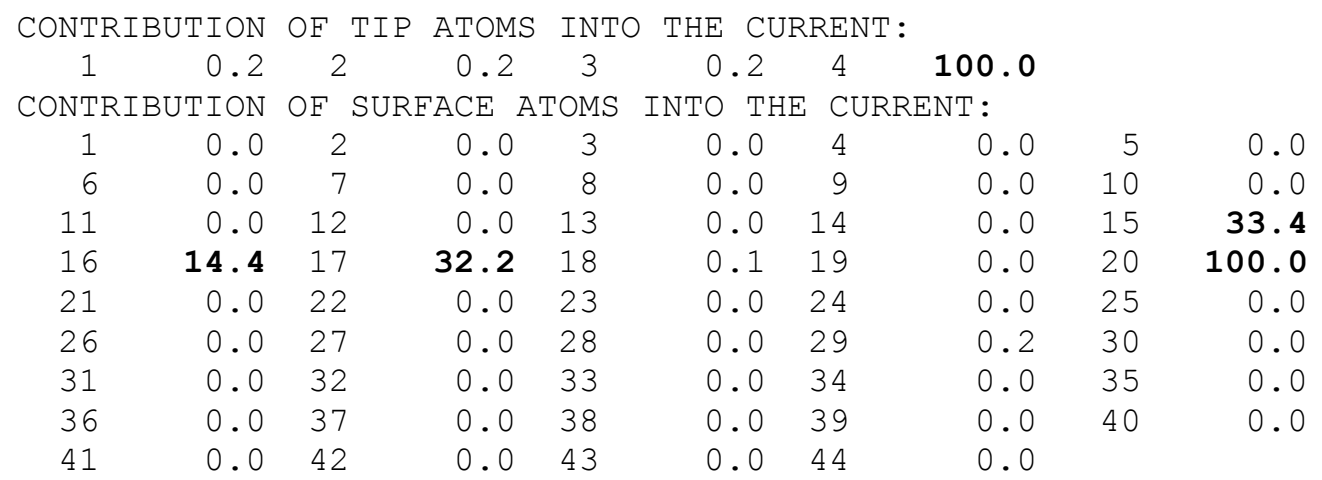

\subsection{Topographic scanning of the tunnel current}

Fig. 6 presents a space contour map of the studied surface obtained by calculating the tip displacement with respect to the surface at a preset value of the tunnel current. The picture corresponds to scanning over 288 points of the $9.20 \times 10.34 \mathrm{~A}^{2}$ grid area with increments of 0.613 and $0.608 \mathrm{~A}$ along $X$ and $Y$ axes, respectively. The obtained picture obviously well reflects the atomic structure shown in Fig.4. The calculation procedure is rather time-consuming and has taken $\sim 40$ hours at PC-Intel PIII, 660MGz. 


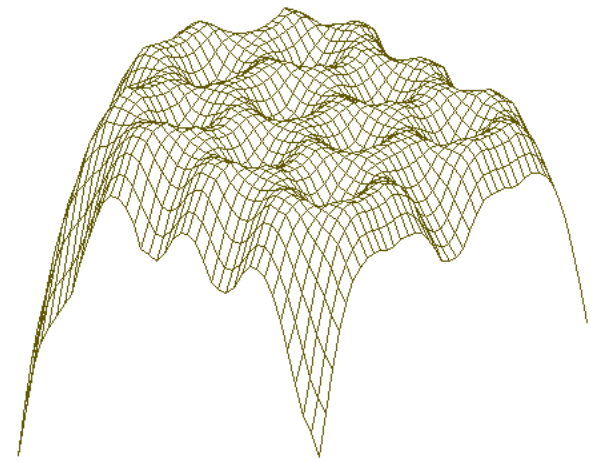

Figure 6. Topographic space image of the surface. The tunnel current is $29.57 \mathrm{pA}$, the surface bias voltage $V=+1 \mathrm{~V}$. HMODE program in action.

\subsection{Current-image of the surface}

The current image of the studied surface is shown in Fig.7. That corresponds to the tunnel current calculation at fixed surface-tip distance. Oppositely to the previous case, the calculations have been performed by scanning over 960 points of the $9.20 \times 10.34 \mathrm{~A}^{2}$ grid area with increments of 0.307 and $0.323 \mathrm{~A}$ along $X$ and $Y$ axes, respectively. As seen from the figure, the obtained picture is well consistent with atomic structure of the surface (see Fig.4) and is characterized by much larger amplitude of the current changing that causes a well pronounced top-view picture shown in Fig. 7b. Regions with high density of lines on the picture correspond to low tunnel current while poorly dense are related to current maxima. The calculation procedure is rather quick and has taken $\sim 20$ hours on the same machine while the number of grid points is 3.33 times more.
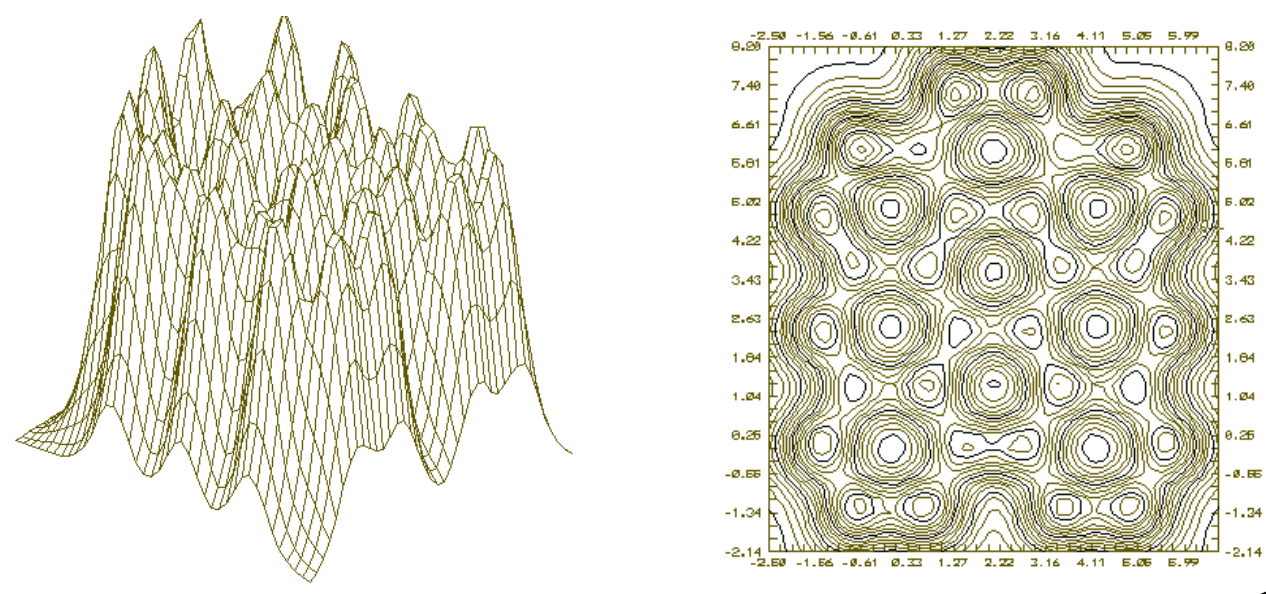

a

Figure 7. Current image of the surface. The surface-tip distance is $2 \mathrm{~A}$, the surface bias voltage $V=+1 \mathrm{~V}$. 960-point grid of $9.20 \times 10.43 \mathrm{~A}^{2}$ in size. IMODE program in action. a. Space view. b. Top view. 


\subsection{Local density of states of the surface}

The calculation procedure for the $\rho_{S T M}(R, V)$ quantity is the least time-consuming so that even modest computational machine can easily provide calculations over manypoint grid with small increments. Fig. 8 shows space and top views of the calculated LDOS over 960 points of the $9.20 \times 10.34 \mathrm{~A}^{2}$ grid area with increments of 0.307 and $0.323 \mathrm{~A}$ along $X$ and $Y$ axes. The picture exactly reproduces the atomic structure of the surface and is of the biggest contrast among the discussed previously. This had to be expected since the integrating procedures that are involved in calculations of either tip displacement or tunnel current smooth the contrast by sure.
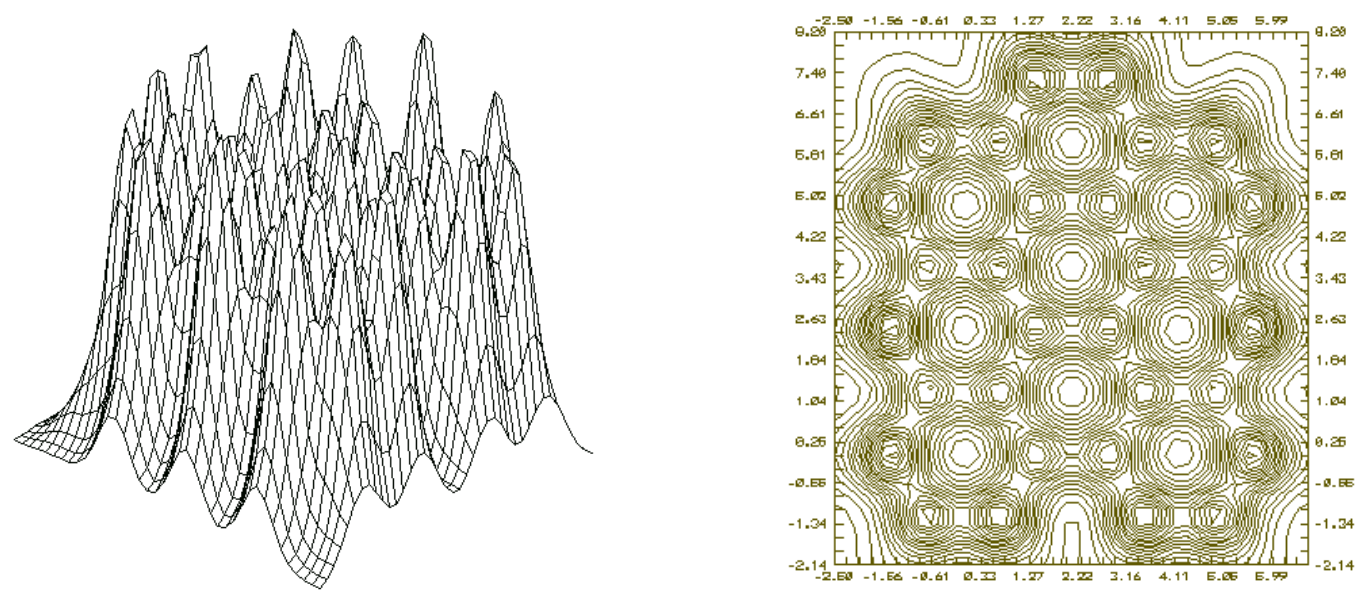

a

Figure 8. Local density $\rho_{S T M}(R, V)$ of the surface at the surface bias voltage $V=+1 \mathrm{~V}$. RoSTM program in action. 960 point grid of $9.20 \times 10.43 \mathrm{~A}^{2}$ in size. a. Space view. $b$. Top view.

\section{Discussion}

A general look at the pictures in Figs. 6, 7, and 8 convincingly evidences towards undoubtedly close similarity both between the pictures themselves as well as between them all and the atomic structure shown in Fig.4. Conveniently, theoretical studies of STM events are stopped on this stage that provides a basis for a qualitative conclusions concerning electron structure of a surface studied. However, computations are always "more pure" experiment, if for no reasons than that real sample treating conditions are excluded from the consideration. That is why a detailed analysis of the computational results allows to penetrate into the depth of the physical event studied much deeper. One of possibilities of such analysis is based on treating scanning results. Multi-mode facilities of the TUNCNR codes calculations form a good ground for such study due to identity of scanning procedure for each computational mode. A comparative analysis will be carried out for scanning over 288-point grid of $9.20 \mathrm{X} 10.43 \mathrm{~A}^{2}$ in size. A general view of scanning results, which is typical for all three computational modes, is presented in Fig.9. Clearly seen in the figure are 16 groups of peaks (scan group below) corresponding to 16 points along $X$ - 
coordinate of the grid. Each group consists of 18 bars in relation to 18 points along $Y$ coordinate of the grid. The picture is symmetric with respect to a reflection plane located between points 144 and 145 in full accordance with a symmetry of the atomic configuration of the surface. Hereinafter, only left part of the relevant pictures will be considered to make comparative study more informative.

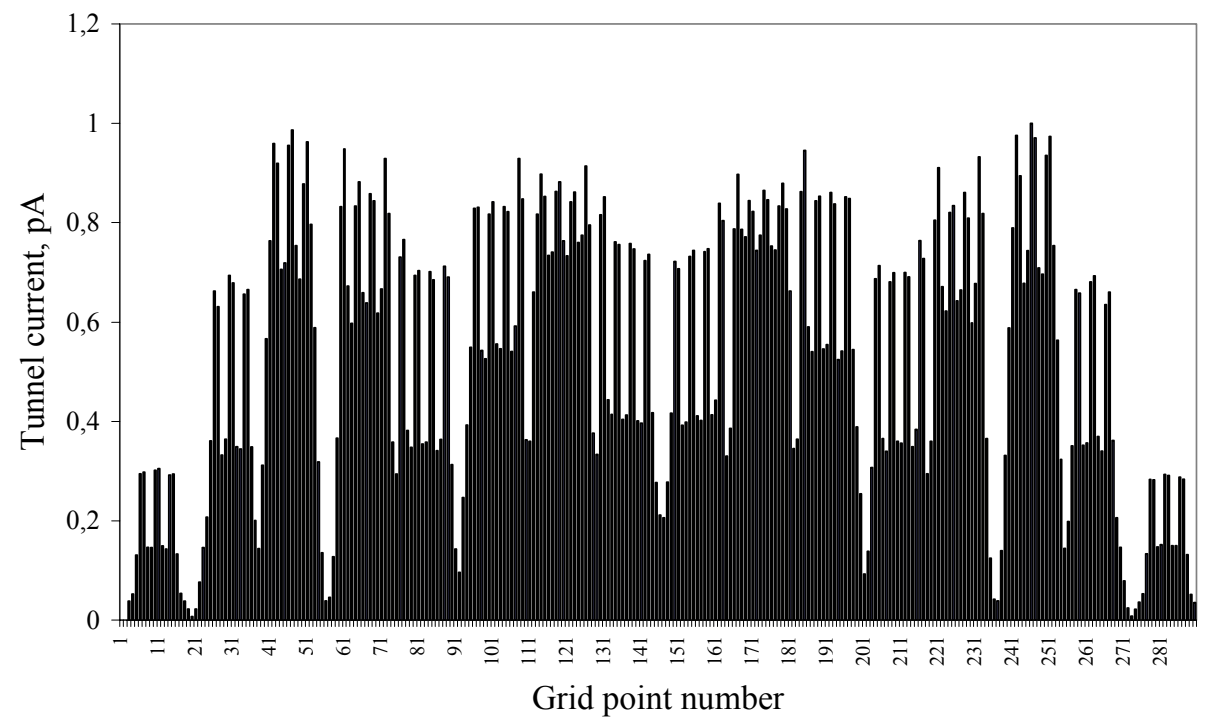

Figure 9. General view of scanning results in the current-image mode. Surface bias voltage $V=+1 \mathrm{~V}$, the tip is kept at $2 \mathrm{~A}$ above the surface. The current values are scaled with respect to maximum one which is set to 1.00 .

\subsection{Bias polarity dependence}

Scanning results related to three computational modes are presented in Fig.10 at two polarities of the surface bias voltage. Substituting a histogram in Fig.9 by continuous curves makes it possible to see the distribution of the computed quantities along the line of scanning by taking into account the "space resolution" of the calculations that is determined by the grid increments.

As seen from the figure, scanning pattern of all three quantities is practically identical. The scan group shape is best exhibited in the case of LDOS in Fig.10c that allows a clear analysis of the correlation between the shape of each group and the atomic structure of the studied surface. The first three groups are of three-peak shape that can be naturally attributed to three hydrogen and three carbon atoms aligned along $Y$-axis at the left side of the surface. The next five groups are of four-peak shape that is consistent with four groups of carbon atoms, each consisting of four atoms. Regular peaks at the bottom of the figure match the location of the surface atoms with respect to the grid points. Since the point coordinates do not coincide with the atom center positions exactly, some discrepancy in the atom positions with respect to maxima of scan groups cannot be avoided. However, the discrepancy is not too big so that a clear conclusion can be made that main peaks of the LDOS are caused by carbon atoms. Four hydrogen atoms in the central part of the surface cluster are distinctly seen as shoulders of the main peaks as well. The calculated results present that very case when the corresponding LDOS and, consequently the tunnel current 


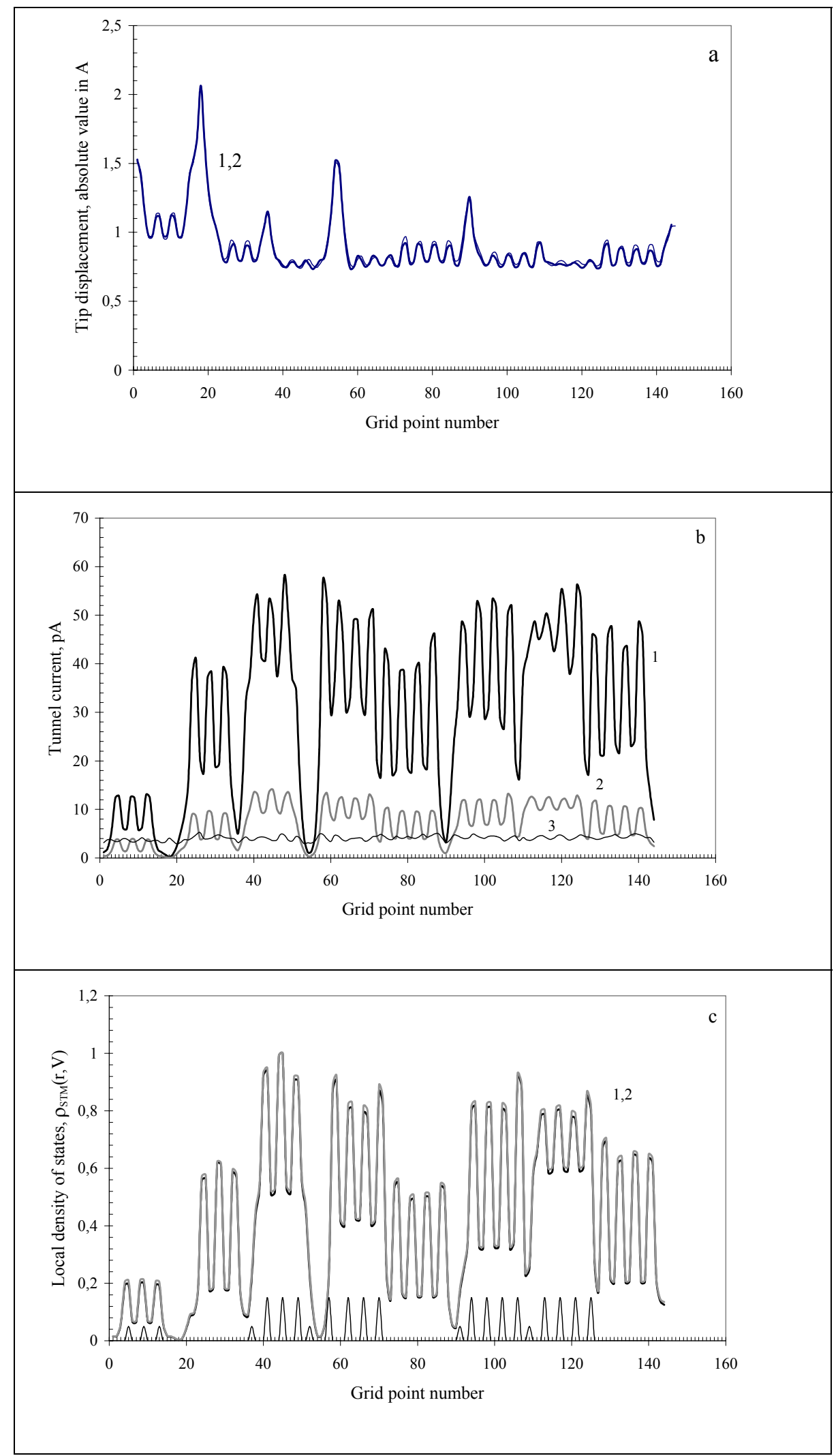

Figure 10. Scan groups at two bias voltages. a. Topographic mode (tunnel current is preset at $29.57 \mathrm{pA}$ ). b. Current-image mode (tip is kept at $2 \mathrm{~A}$ above the surface). c. LDOS $\rho_{S T M}(R, V)$. Curves 1 and 2 are related to surface bias voltages of $+1 \mathrm{~V}$ and 
$-1 \mathrm{~V}$, respectively. Curve 3 presents the ratio of data in curves 1 and 2 . High and low regular peaks match positions of carbon and hydrogen atoms, respectively.

and the tip displacement exhibit real atomic structure of the surface. Alternative situations which related to simulations of real surfaces will be considered elsewhere [21]. Since the increment along the $X$-axes is less than the distance between carbon atoms, the number of scan groups exceeds that of atoms. When going from the LDOS to the tunnel current and the tip displacement, the structure of the scan groups remains the same by shape but becomes less and less contrast due to integrating nature of the tunnel current.

The results in Fig.10 are given for two polarities of the surface bias, equal by magnitude. As seen, changing polarity does not effect both the LDOS and the tip displacement distributions, so that curves 1 and 2 are practically non-distinguishable. The main observation for the tunnel current shown in Fig.10b, concerns almost four times decreasing of the current at negative polarity. Curve 3 in the figure shows that this factor is quite steadily kept within all scans.

\subsection{LDOS and topographic and current images of the surface}

As follows from the above analysis, a comparison on the level of scan groups convincingly proves a good consistence between the $\rho_{S T M}(R, V)$ and STM recording in both modes, including not only general features but the distribution details as well. Continuing a comparison on the level, Fig.11 shows this consistence in more details. As seen from Fig.11a, in the case of the current-image mode, fitting between the LDOS and the tunnel current is not only qualitative, but quantitative to a great extent. Both curves in the figure are given in the same scale. As seen in the figures all peaks of the LDOS are bigger, though in different ratio for different scan groups, while minima well coincide with those for the tunnel current. The feature causes a visual damping of the contrast of picture in Fig.7 in comparison with those in Fig.8. It should be noted, however, that presented results evidently witness against the TersoffHamann relation $I(R)=V \rho\left(R, E_{F}\right)[\mathrm{TH}]$ since no linearity between the two values has been observed. The connection seems to be much more complex and individual with respect to different surface-tip systems.

In the case of topographic mode (Fig.11b), there a strict correlation in the positions of the least tip displacements (or the highest tip positions) and the LDOS peaks. Therefore, the picture shown in Fig.6 reflects the atomic structure of the surface. However, amplitudes of the tip position variation are much less than those of LDOS that drastically smoothes the contrast of the recording picture.

\section{Conclusion}

TUNCUR codes are aimed at a coherent calculation of both equilibrated structures and electronic properties of a surface and STM tip altogether with the tunneling current between them on the same computational footing. Both objects are modeled by atomic clusters. TUNCUR is based on sequential codes CLUSTER-Z1 and CLUSTER-Z2 that provide SCF HF calculations in the valence $s p$ - и $s p d$-basis, respectively. The calculations supply further procedures with eigen values and eigen 
states of the substrate and tip The codes two big program blocks that provide the performance of calculations which are adequate to a topographic (the current is fixed and the displacement of the tip position is determined, HMODE) and current-image (the tip position high is fixed and the tunnel current is calculated, IMODE) modes of conventional STM operation. Additionally to the above STM recordings, the block RoSTM do calculations of the local density of states $\rho_{S T M}(R, V)$ near the Fermi energy region. The latter provides both an internal checking of the calculations
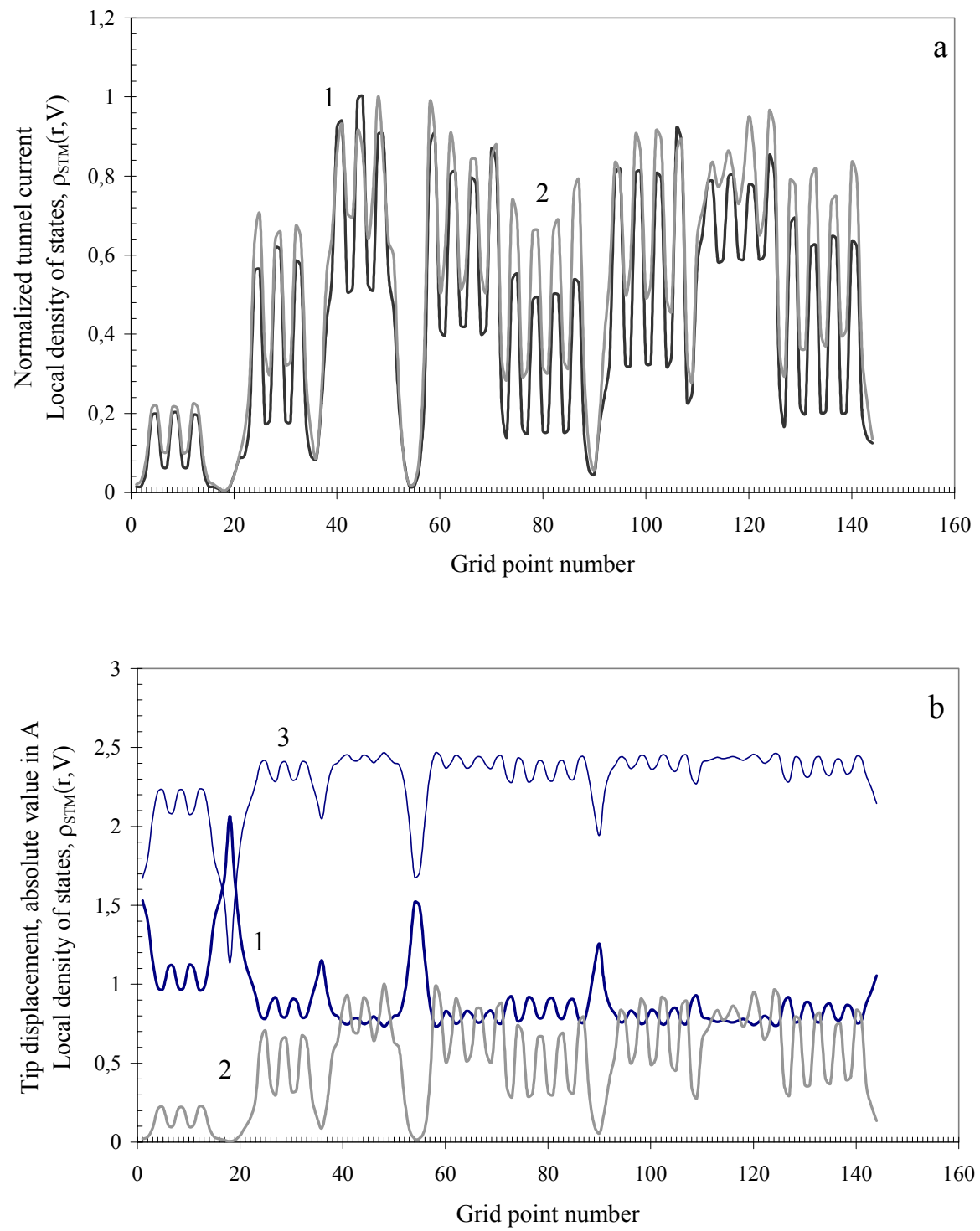

Figure 11. Correlation between LDOS $\rho_{S T M}(R, V)$ (curves 1) and STM recordings at the current-image ( $a$, tip is kept at $2 \mathrm{~A}$ above the surface) and topographic ( $b$, the tunnel current is preset at $29.57 \mathrm{pA}$ ) modes of operation. Curves 1 correspond to the scaled tunnel current (a) and tip displacement (b). Curve 3 presents the tip position over surface during the scans. Surface bias voltage $V=+1 \mathrm{~V}$. 
performed as well as exhibiting a vivid correlation between the STM recordings and electronic properties of the substrate. All calculations are performed over grid, the number of points of which as well its linear dimensions and, consequently, increments along grid axes are input from the screen.

The code facility approbation has been performed for a virtual model of the surface that is presented by a planar configuration of nine benzene rings. A cluster of four Ti atoms simulated the tip. The results have been analyzed by both comparing space and planar contour maps and considering scanning data quantitatively. Keeping identical scanning conditions forms the ground for a few conclusions, important from both theoretical and experimental viewpoint.

1. A widely exploited theoretical relation of Tersoff and Hamann $I(R)=V \rho\left(R, E_{F}\right)$ occurred to be not quantitatively valid and the dependence between the tunnel current and LDOS is more complex.

2. In spite of the fact, there is a strong correlation between features of space distribution of the LDOS, on the one hand, and either tunnel current, or tip displacement, on the other.

3. Integrating that is incorporated in the tunnel current calculation, inevitably causes a considerable smoothing of the space pattern contrast, decreasing amplitudes of the two quantities variation.

4. Between two modes of STM operation, a topographic one is much less contrast.

The codes efficiency is high enough to recommend them for use in convenient STM studies. Further implementation of parallel computing into the codes will result in significant speed-up of calculations.

The project is fulfilled in the framework of grant № 40.012.1.1.1152 of the Ministry of Industry, Science and Technology of the Russian Federation

\section{References}

1. Binnig, G., Rohrer, H., Gerber, Ch., Weibel, E. Phys.Rev.Letts. 49 (1982) 57.

2. Bryant, A., Smith, D.P.E., Quate, C.F. Appl.Phys.Letts. 48 (1986) 832.

3. Tsukada, M., Kobayashi, K., Isshiki, N., Kageshima, H. Surf.Sci.Rep. 13 (1991) 265.

4. Briggs, G.A.D., Fisher, A.J. Surf.Sci.Rep. 33 (1999) 1.

5. Tsukada, M., Shima, N. J.Phys.Soc.Jpn. 56 (1987) 2875.

6. Tsukada, M., Kobayashi, K., Isshiki, N., Watanabe, S., Kageshima, H., Schimuzu, T. J.Mol.Cat., 82 (1993) 253.

7. Badziag, P., Verwoerd, W.S. Surf.Sci. 285 (1993) 145.

8. Tsukada, M., Kobayashi, K., Ohnishi, S. J.Vac.Sci.Technol. A8 (1990) 160.

9. Zayets, V.A. CLUSTER-Z1: Quantum-Chemical Software for Calculations in the $s, p$-Basis: Institute of Surface Chemistry, Nat. Ac.Sci. of Ukraine: Kiev (1990).

10.Zayets, V.A. CLUSTER-Z2: Quantum-Chemical Software for Calculations in the $s, p, d$-Basis: Institute of Surface Chemistry, Nat. Ac.Sci. of Ukraine: Kiev (1991).

11.Forsythe, G.,E., Malcolm, M., A., Moler, C., B. Computer Methods For Mathematical Computations, Prentice-Hall Inc., Engle Wood Clyffs, N.J. 1977.

12. Tersoff, J., Hamann, D.,R. Phys.Rev.Letts. 50 (1983) 1998; Phys.Rev. B31 (1985) 805.

13. Tomanek, D., Louie, S. Phys.Rev. 37 (1988) 8327. 
14. Grebenjuk A.G. ,Zayets V.A. ,Gorlov Yu.I. , React. Kinet. Catal. Lett. ,50 No. $1 / 2(1993) 257$.

15. Zayets V.A. .Gerda V.I. ,Gorlov Yu.I. ,Klimenko V.E. , Zh. Struct. Khim. ,39 No. 1 (1998) 163.

16. Zayets V.A. .Gerda V.I. ,Gorlov Yu.I. ,Klimenko V.E., Zh. Struct. Khim. ,40 No. 1 (1998) 111.

17. Zayets V.A. Gerda V.I. Zh. Struct. Khim. 43 No. 2 (2002) 372.

18. Berzigiyarov, P.K., Zayets, V.A., Ginzburg, I.Ya., Razumov, V.F., Sheka, E.F. TASK Quarterly 6 (2002) 233; Int.Journ.Quant.Chem. 88 (2002) 449.

19. Zayets, V., A. NDDO-WF parameters of Ti atoms, Kiev, 2001.

20. Fujita, D., Sagisaka, K., Kitahara, M., Ohgi, T. Phys. Low-Dim.Struct, No.3/4 (2003) 167

21. Sheka, E., F., Zayets, V., A., to be published. 